\title{
Exact analysis of resonance frequency and mode shapes of isotropic and laminated composite cylindrical shells; Part I: analytical studies ${ }^{\dagger}$
}

\author{
Pouria Oliazadeh ${ }^{1, *}$, Mohammad H. Farshidianfar ${ }^{2}$ and Anooshiravan Farshidianfar ${ }^{1}$ \\ ${ }^{1}$ Mechanical Engineering Department, Ferdowsi University of Mashhad, Mashhad, Iran \\ ${ }^{2}$ Mechanical Engineering Department, University of Waterloo, Waterloo, Canada
}

(Manuscript Received January 29, 2013; Revised June 8, 2013; Accepted July 2, 2013)

\begin{abstract}
In order to study the free vibration of simply supported circular cylindrical shells, an exact analytical procedure is developed and discussed in detail. Part I presents a general approach for exact analysis of natural frequencies and mode shapes of circular cylindrical shells. The validity of the exact technique is verified using four different shell theories 1) Soedel, 2) Flugge, 3) Morley-Koiter and 4) Donnell. The exact procedure is compared favorably with experimental results and those obtained using a numerical finite element method. A literature review reveals that beam functions are used extensively as an approximation for simply supported boundary conditions. The accuracy of the resonance frequencies obtained using the approximate method are also investigated by comparing results with those of the exact analysis. Part II presents effects of different parameters on mode shapes and natural frequencies of circular cylindrical shells.
\end{abstract}

Keywords: Circular cylindrical shell; Exact approach; Numerical method; Natural frequency

\section{Introduction}

Similar to beams and plates, cylindrical shells are the practical elements of various engineering structures such as pipes and ducts, car bodies, space shuttles, aircraft fuselages, ship hulls, submarines, and buildings. However, analyzing the dynamic characteristics of cylindrical shells is more complicated than analyzing those of beams and plates. This situation occurs mainly because the equations of motion in a cylindrical shell are more complicated. Moreover, in many cases boundary condition effects are hard to apply.

A comprehensive summary and discussion of shell theories, including natural frequencies and mode shape identification, has been done by Liessa [1] in 1973. More recently, adopting a non-linear point of view, Amabili and Paidoussis [2], Amabili [3] and Kurylov and Amabili [4] have presented noteworthy reviews on cylindrical shell vibration. Many researchers such as Flugge [5] have followed the pioneering work of Love [6], using his first approximation theory. The Flugge theory is based on the Kirchhoff-Love hypothesis for thin elastic shells. Using this theory, the strain-displacement relations and changes of curvature in the middle surface of a cylindrical shell can be obtained. The simplified Donnell's theory is achieved by neglecting a few terms in the Flugge

*Corresponding author. Tel.: +98 5118763304, Fax.: +98 5118763304

E-mail address: pouria.oliazadeh@gmail.com

${ }^{\dagger}$ Recommended by Editor Yeon June Kang

(C) KSME \& Springer 2013 equations.

Livanov [7] applied love's assumption and used displacement functions to solve the problem of axisymmetrical vibrations of simply supported cylindrical shells. Rinehart and Wang [8] investigated the vibration of simply supported cylindrical shells stiffened by discrete longitudinal stiffeners using Donnell's approximate theory, Flugge's exact theory, and Love's assumption for longitudinal wave numbers. These theories are not only suitable for simply supported end conditions, but they can also be applied to other cylindrical shell boundary conditions such as fixed free [9, 10], clampedclamped [11] and infinite length ones [12]. In most reports available in the literature, beam functions are used as approximations for the boundary condition effects. Thus, resonance frequencies are obtained through an approximate procedure rather than an exact analysis. The main reason for these approximations is the difficulties posed when actual boundary conditions are applied.

The first part of the present study proposes an exact analytical approach to investigate the free vibration of simply supported cylindrical shells. As mentioned, in conventional analysis, beam functions with similar boundary conditions are used to approximate wave numbers in the axial direction. This approach is considered to be approximate; however, the degree of uncertainty in these approximations is unknown. An exact method is developed, which will consider the actual effects of shell boundaries in order to obtain exact resonance 


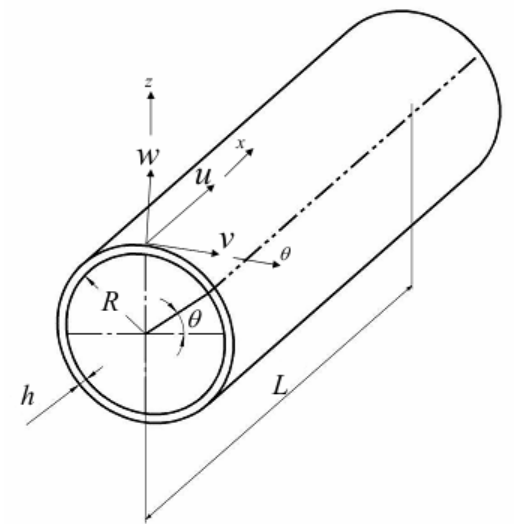

Fig. 1. Circular cylindrical shell: coordinate system and dimensions.

frequencies of a cylindrical shell. The exact method is used to obtain the natural frequencies based on four different shell theories (Soedel, Flugge, Morley-Koiter and Donnell). This method is compared with the approximate method, to study the validity of beam function approximations. The results are also compared with experimental and numerical ones and show good agreement.

\section{Theoretical analysis}

As mentioned in the literature, circular cylindrical shells with both ends closed have wide applications in the aviation industry. Moreover, hemispherical shells closed at both ends are broadly used for pressure vessels and storage tanks [13].

A schematic diagram of a circular cylindrical shell closed at both ends is given in Fig. 1. The cylindrical shell under consideration has a constant thickness $h$, mean radius $R$, axial length $L$, Poisson's ratio $v$, density $\rho$ and Young's modulus of elasticity $E$. Here, the respective displacements in the axial, circumferential and radial directions are denoted by $u(x, \theta, t)$, $v(x, \theta, t)$ and $w(x, \theta, t)$.

In order to study free vibration of a cylindrical shell, the equations of motion can be written in matrix form as follows:

$$
\left[\begin{array}{ccc}
L_{11} & L_{12} & L_{13} \\
L_{12} & L_{22} & L_{23} \\
-L_{13} & -L_{23} & L_{33}
\end{array}\right]\left\{\begin{array}{c}
u(x, \theta, t) \\
v(x, \theta, t) \\
w(x, \theta, t)
\end{array}\right\}=\left\{\begin{array}{l}
0 \\
0 \\
0
\end{array}\right\}
$$

where $L_{i j}(i, j=1,2,3)$ are differential operators with respect to $x, \theta$ and $t$.

Different systems of equations are used to model the vibration behavior of circular cylindrical shells. In this paper four of the most common theories, namely: 1) Soedel [14], 2) Flugge [5], 3) Morley-Koiter [15] and 4) Donnell's theory [3], are used to find natural frequencies. It is convenient to define the following differential operators for these theories, respectively:

$$
\begin{aligned}
& L_{11}=-\frac{\rho}{E}\left(1-v^{2}\right) \frac{\partial^{2}}{\partial t^{2}}+\frac{\partial^{2}}{\partial x^{2}}+\frac{1-v}{2 R^{2}} \frac{\partial^{2}}{\partial \theta^{2}} \\
& L_{12}=\frac{1+v}{2 R} \frac{\partial^{2}}{\partial x \partial \theta}, L_{13}=\frac{v}{R} \frac{\partial}{\partial x} \\
& L_{22}=-\frac{\rho}{E}\left(1-v^{2}\right) \frac{\partial^{2}}{\partial t^{2}}+(1+k)\left(\frac{1-v}{2} \frac{\partial^{2}}{\partial x^{2}}+\frac{1}{R^{2}} \frac{\partial^{2}}{\partial \theta^{2}}\right) \\
& L_{23}=\frac{\partial}{\partial \theta}\left[\frac{1}{R^{2}}-k\left(\frac{\partial^{2}}{\partial x^{2}}+\frac{1}{R^{2}} \frac{\partial^{2}}{\partial \theta^{2}}\right)\right] \\
& L_{33}=-\frac{\rho}{E}\left(1-v^{2}\right) \frac{\partial^{2}}{\partial t^{2}}-\left[\frac{1}{R^{2}}+k\left(R \frac{\partial^{2}}{\partial x^{2}}+\frac{1}{R} \frac{\partial^{2}}{\partial \theta^{2}}\right)^{2}\right] \\
& L_{11}=-\frac{\rho}{E}\left(1-v^{2}\right) \frac{\partial^{2}}{\partial t^{2}}+\frac{\partial^{2}}{\partial x^{2}}+(1+k) \frac{1-v}{2 R^{2}} \frac{\partial^{2}}{\partial \theta^{2}} \\
& L_{12}=\frac{1+v}{2 R} \frac{\partial^{2}}{\partial x \partial \theta} \\
& L_{13}=\frac{v}{R} \frac{\partial}{\partial x}+k\left(\frac{1-v}{2 R} \frac{\partial^{3}}{\partial x \partial \theta^{2}}-R \frac{\partial^{3}}{\partial x^{3}}\right) \\
& L_{22}=-\frac{\rho}{E}\left(1-v^{2}\right) \frac{\partial^{2}}{\partial t^{2}}+(1+2 k)\left(\frac{1-v}{2}\right)+\frac{1}{R^{2}} \frac{\partial^{2}}{\partial \theta^{2}} \\
& L_{23}=\frac{1}{R^{2}} \frac{\partial}{\partial \theta}-k\left(\frac{3-v}{2}\right) \frac{\partial^{3}}{\partial x^{2} \partial \theta} \\
& L_{33}=-\frac{\rho}{E}\left(1-v^{2}\right) \frac{\partial^{2}}{\partial t^{2}} \\
& -\left[\frac{1}{R^{2}}+k\left(1+\left(R \frac{\partial^{2}}{\partial x^{2}}+\frac{1}{R} \frac{\partial^{2}}{\partial \theta^{2}}\right)^{2}+\frac{2}{R^{2}} \frac{\partial^{2}}{\partial \theta^{2}}\right)\right] \\
& L_{11}=-\frac{\rho}{E}\left(1-v^{2}\right) \frac{\partial^{2}}{\partial t^{2}}+\frac{\partial^{2}}{\partial x^{2}}+\frac{1-v}{2 R^{2}} \frac{\partial^{2}}{\partial \theta^{2}} \\
& L_{12}=\frac{1+v}{2 R} \frac{\partial^{2}}{\partial x \partial \theta}, L_{13}=\frac{v}{R} \frac{\partial}{\partial x} \\
& L_{22}=-\frac{\rho}{E}\left(1-v^{2}\right) \frac{\partial^{2}}{\partial t^{2}}+\frac{1-v}{2} \frac{\partial^{2}}{\partial x^{2}}+\frac{1}{R^{2}} \frac{\partial^{2}}{\partial \theta^{2}} \\
& L_{23}=\frac{1}{R^{2}} \frac{\partial}{\partial \theta}, L_{33}=-\frac{\rho}{E}\left(1-v^{2}\right) \frac{\partial^{2}}{\partial t^{2}} \\
& -\left\{\frac{1}{R^{2}}+k\left[\left(R \frac{\partial^{2}}{\partial x^{2}}+\frac{1}{R} \frac{\partial^{2}}{\partial \theta^{2}}\right)^{2}+\frac{1}{R^{2}}\left(2 \frac{\partial^{2}}{\partial \theta^{2}}+1\right)+2 \frac{\partial^{2}}{\partial x^{2}}\right]\right\} \\
& L_{11}=-\frac{\rho}{E}\left(1-v^{2}\right) \frac{\partial^{2}}{\partial t^{2}}+\frac{\partial^{2}}{\partial x^{2}}+\frac{1-v}{2 R^{2}} \frac{\partial^{2}}{\partial \theta^{2}} \\
& L_{12}=\frac{1+v}{2 R} \frac{\partial^{2}}{\partial x \partial \theta}, L_{13}=\frac{v}{R} \frac{\partial}{\partial x} \\
& L_{22}=-\frac{\rho}{E}\left(1-v^{2}\right) \frac{\partial^{2}}{\partial t^{2}}+\frac{1-v}{2} \frac{\partial^{2}}{\partial x^{2}}+\frac{1}{R^{2}} \frac{\partial^{2}}{\partial \theta^{2}} \\
& L_{23}=\frac{1}{R^{2}} \frac{\partial}{\partial \theta} \\
& L_{33}=-\frac{\rho}{E}\left(1-v^{2}\right) \frac{\partial^{2}}{\partial t^{2}}-\left[\frac{1}{R^{2}}+k\left(R \frac{\partial^{2}}{\partial x^{2}}+\frac{1}{R} \frac{\partial^{2}}{\partial \theta^{2}}\right)^{2}\right]
\end{aligned}
$$


The operators in Eqs. (2)-(5) can be treated as the sum of two operators as done by Leissa [1]:

$$
\mathbf{L}=\mathbf{L}_{\mathbf{D}}+k \mathbf{L}_{\mathbf{M O D}}
$$

where $\mathbf{L}_{\mathbf{D}}$ is the differential operator according to the Donnell theory. $\mathbf{L}_{\mathbf{M O D}}$ is a modifying operator that differs for each theory and is presented in Appendix A. Moreover, $k$ is the nondimentional thickness parameter defined by

$$
k=\frac{h^{2}}{12 R^{2}}
$$

The first attempt at solving Eq. (1) involves the assumption of a synchronous motion:

$$
\left\{\begin{array}{l}
u(x, \theta, t)=U(x, \theta) f(t) \\
v(x, \theta, t)=V(x, \theta) f(t) \\
w(x, \theta, t)=W(x, \theta) f(t)
\end{array}\right.
$$

where $f(t)$ is the scalar model coordinate corresponding to the mode shapes $U(x, \theta), V(x, \theta)$ and $W(x, \theta)$.

The next step is to use the separation of variables method in order to separate the spatial dependence of the modal shape between longitudinal and circumferential directions. Hence, the axial, tangential and radial displacements of the wall vary according to:

$$
\left\{\begin{array}{l}
u(x, \theta, t)=A e^{\lambda_{m} x} \sin (n \theta) \cos (\omega t) \\
v(x, \theta, t)=B e^{\lambda_{m} x} \cos (n \theta) \cos (\omega t) \\
w(x, \theta, t)=C e^{\lambda_{m} x} \sin (n \theta) \cos (\omega t)
\end{array}\right.
$$

in which $\lambda_{m}$ and $n$ are the axial wavenumber and the circumferential wave parameter, respectively. $A, B$ and $C$ are the undetermined constants, and $\omega$ is the circular frequency of the natural vibration.

Substituting Eq. (9) into Eq. (1), using any of the shell theories given by Eqs. (2)-(5), leads to a set of homogenous equations having the following matrix form:

$$
\left[\begin{array}{ccc}
C_{11} & C_{12} & C_{13} \\
-C_{12} & C_{22} & C_{23} \\
-C_{13} & C_{23} & C_{33}
\end{array}\right]\left\{\begin{array}{l}
A \\
B \\
C
\end{array}\right\}=\left\{\begin{array}{l}
0 \\
0 \\
0
\end{array}\right\}
$$

in which $\left[C_{i j}\right](i, j=1,2,3)$ are functions of $n, \lambda_{m}$ and a frequency parameter $\Omega$ that is defined as follows:

$$
\Omega^{2}=\frac{\left(1-v^{2}\right) \rho}{E} \omega^{2} R^{2}
$$

As an example, for Donnell's theory, $\left[C_{i j}\right]$ can be written in matrix form as in Eq. (12). The coefficient matrix for other shell theories is defined in Appendix B.

$$
\left[\begin{array}{ccc}
\Omega^{2}+\lambda_{m}^{2}-\frac{1-v}{2} n^{2} & -\frac{1+v}{2} n \lambda_{m} & v \lambda_{m} \\
\frac{1+v}{2} n \lambda_{m} & \Omega^{2}+\frac{1-v}{2} \lambda_{m}^{2}-n^{2} & n \\
-v \lambda_{m} & n & \Omega^{2}-\left[1+k\left(\lambda_{m}^{2}-n^{2}\right)^{2}\right]
\end{array}\right]
$$

For a nontrivial solution, the determinant of the coefficient matrix in Eq. (10) must be zero:

$$
\operatorname{det}\left(\left[C_{i j}\right]\right)=0 ; i, j=1,2,3 \text {. }
$$

The expansion of Eq. (13) will give the following two eigenvalue problems:

- For a given value of $\lambda_{m}$, there exists one or more proper values for $\omega$ so that Eq. (13) vanishes.

- For a given value of $\omega$, there exists one or more proper values for $\lambda_{m}$ so that Eq. (13) vanishes.

Solving Eq. (13) leads to a cubic equation in terms of the non-dimensional frequency parameter $\Omega^{2}$. Thus, for a fixed value of $n$ and $\lambda_{m}$, three positive roots and three negative roots are yielded for the non-dimensional frequency. The three positive roots are the natural frequencies of the cylindrical shell that can be classified as primarily axial, circumferential or radial. The lowest frequency is usually associated with a motion that is primarily radial (or flexural).

\subsection{The approximate beam function method}

In general, solving the roots of the characteristic equation of Eq. (13) for $\lambda_{m}$ is not possible in closed form. Hence, researchers have often favored towards using approximate techniques. According to previous studies, beam functions are widely used to obtain natural frequencies and approximate displacements for closed circular cylindrical shells. According to the approximate method, for a shell simply supported at both ends, the nature of the axial mode can be defined as:

$$
\lambda_{m}=m \pi \frac{R}{L} \sqrt{-1}
$$

Substituting Eq. (14) into Eq. (13), the only unknown of the characteristic equation will be the frequency parameter $\Omega^{2}$ for a fixed combination of $m$ and $n$. For cylindrical shells with simply supported boundary conditions, typical flexural, longitudinal and circumferential nodal patterns according to different $m$ and $n$ combinations are shown in Fig. 2.

Although this approach is straightforward, it is an approximation for boundary conditions of a simply supported circular cylindrical shell. However, since cylindrical shell vibration is totally different compared to that of beams, it is important to check the accuracy of this approximation.

Thus, an exact analytical technique that uses the boundary 


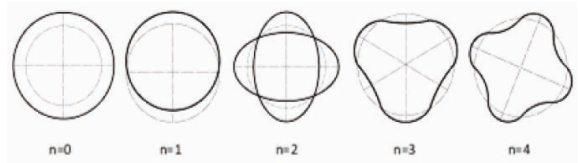

(a)

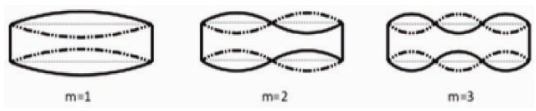

(b)

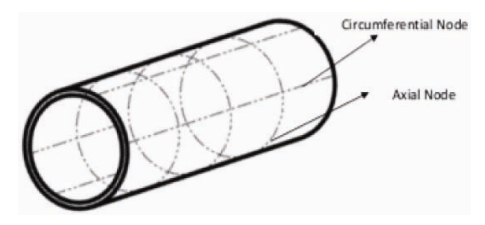

(c)

Fig. 2. Mode shapes of a cylindrical shell: (a) circumferential mode shapes; (b) longitudinal and radial mode shapes; (c) nodal arrangement of a cylindrical shell for $n=2, m=4$.

condition equations to obtain resonance frequencies is proposed. Results of both the approximate and exact methods are compared with experimental and numerical data.

\subsection{The exact method}

This section presents an exact analysis of the vibration of cylindrical shells. The method can be applied to any of the theories discussed.

At each end of the cylindrical shell, four boundary conditions must be specified. For the simply supported shell the following boundary conditions are imposed:

$$
w=0, v=0, \quad M_{x}=0, N_{x}=0 \quad \text { at } \quad x=0, L .
$$

These conditions restrain the $v$ and $w$ components of shell displacements at their mutual boundaries and will cause negligible internal bending moment $M_{x}$ and membrane normal force $N_{y}$ in the shell as the shell deforms. Using the approach mentioned in Ref. [3] and eliminating the nonlinear terms, the boundary condition equations for a simply supported shell are obtained as follows for Soedel [14], Flugge [5], Morley-Koiter [15] and Donnell's theory [3], respectively:

$$
\begin{aligned}
& M_{x}=-\frac{E h^{3}}{12\left(1-v^{2}\right)}\left\{\frac{\partial^{2} w}{\partial x^{2}}+\frac{v}{R^{2}}\left(\frac{\partial^{2} w}{\partial \theta^{2}}-\frac{\partial v}{\partial \theta}\right)\right\} \\
& N_{x}=\frac{E h}{1-v^{2}}\left\{\frac{\partial u}{\partial x}+\frac{v}{R}\left(\frac{\partial v}{\partial \theta}+w\right)\right\} \\
& M_{x}=-\frac{E h^{3}}{12\left(1-v^{2}\right)}\left\{\frac{\partial^{2} w}{\partial x^{2}}+\frac{v}{R^{2}}\left(\frac{\partial^{2} w}{\partial \theta^{2}}-\frac{\partial v}{\partial \theta}\right)-\frac{1}{R} \frac{\partial u}{\partial x}\right\} \\
& N_{x}=\frac{E h}{1-v^{2}}\left\{\frac{\partial u}{\partial x}+\frac{v}{R}\left(\frac{\partial v}{\partial \theta}+w\right)-k R \frac{\partial^{2} w}{\partial x^{2}}\right\}
\end{aligned}
$$

$$
\begin{aligned}
& M_{x}=-\frac{E h^{3}}{12\left(1-v^{2}\right)}\left\{\frac{\partial^{2} w}{\partial x^{2}}+\frac{v}{R^{2}} \frac{\partial^{2} w}{\partial \theta^{2}}+\frac{1-v}{R^{2}} \frac{\partial v}{\partial \theta}+\frac{w}{R^{2}}\right\} \\
& N_{x}=\frac{E h}{1-v^{2}}\left\{\frac{\partial u}{\partial x}+\frac{v}{R}\left(\frac{\partial v}{\partial \theta}+w\right)-k \frac{1-v}{R} \frac{\partial^{2} w}{\partial \theta^{2}}\right\} \\
& M_{x}=-\frac{E h^{3}}{12\left(1-v^{2}\right)}\left\{\frac{\partial^{2} w}{\partial x^{2}}+\frac{v}{R^{2}} \frac{\partial^{2} w}{\partial \theta^{2}}\right\} \\
& N_{x}=\frac{E h}{1-v^{2}}\left\{\frac{\partial u}{\partial x}+\frac{v}{R}\left(\frac{\partial v}{\partial \theta}-w\right)\right\} .
\end{aligned}
$$

Substituting the modal displacements into these constraints, leads to a set of eight homogenous equations, expressed as follows:

$$
[\mathbf{H}]_{8 \times 8}\{\mathbf{b}\}_{8 \times 1}=\{0\}
$$

in which $\omega, \lambda_{m}$ and $b_{i}(i=1, \ldots, 8)$ are the ten unknowns. For a nontrivial solution of Eq. (20) one requires

$$
\operatorname{det}[\mathbf{H}]=0 \text {. }
$$

The driving frequencies are obtained by simultaneously solving both the characteristic equations for the $3 \times 3$ displacement coefficient matrix, Eq. (13), and the $8 \times 8$ boundary condition determinant, Eq. (21).

\section{Results and discussion}

\subsection{Validation of the exact analysis}

A Matlab program was written to obtain the resonance frequencies of a shell, using the exact method described in the previous section. First, exact resonance frequencies were obtained based on the Soedel theory. Consider a cylindrical shell with dimensions of $L / R=3, h / R=1 / 20$ and $v=1 / 3$. In order to yield the exact frequencies, one has to solve Eq. (21) in terms of the non-dimensional frequency parameter, $\Omega$. In Figs. 3-7, the determinant of the boundary coefficient matrix (Eq. (21)) is calculated for constant values of circumferential wave parameter $(n=1,3,5,7,9)$. As pointed out in section 2.2, to obtain a nontrivial solution, the boundary coefficient determinant should be equal to zero at the resonance frequency, $\Omega$. However, as it can be seen by Figs. 3-7, none of the determinants reach zero. This fact is completely explainable considering the sensitivity of Eq. (21) to $\Omega$. To obtain the determinant of the boundary coefficient matrix in Figs. 3-7, a frequency sweep was carried out using steps of $\Delta \Omega=0.001$. On the other hand, Eq. (21) is very sensitive to frequency variations. Thus, although the determinants represented in Figs. 3-7 should actually equal to zero at resonance frequencies, since the frequency steps are not small enough, they may not make the determinant exactly zero. In Figs. 3-7, the determinants are not exactly zero at any frequencies, but several minimum 


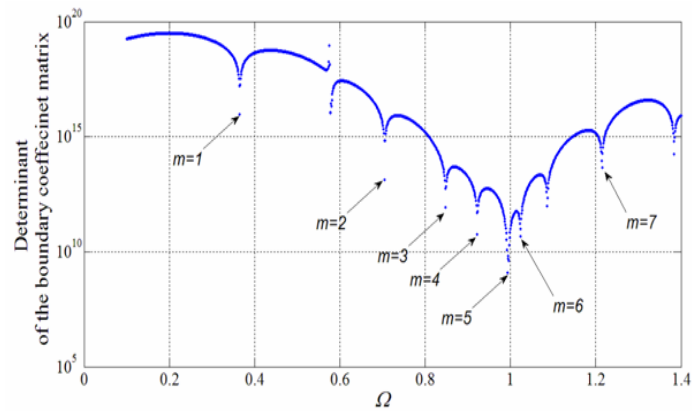

Fig. 3. Determinant of the boundary coefficient matrix versus frequency parameter for $n=1$.

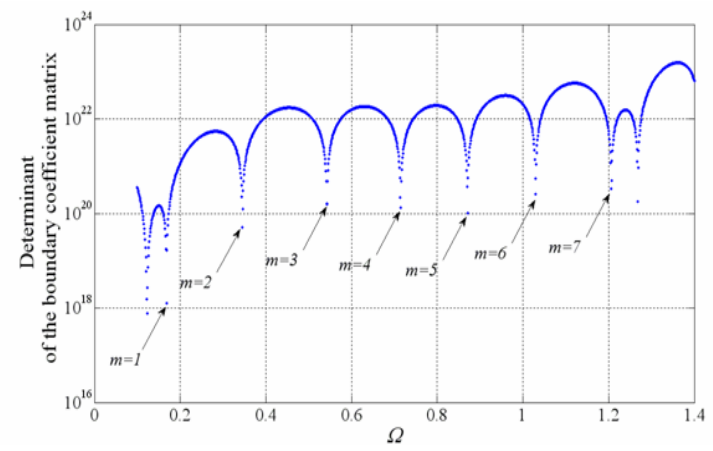

Fig. 4. Determinant of the boundary coefficient matrix versus frequency parameter for $n=3$.

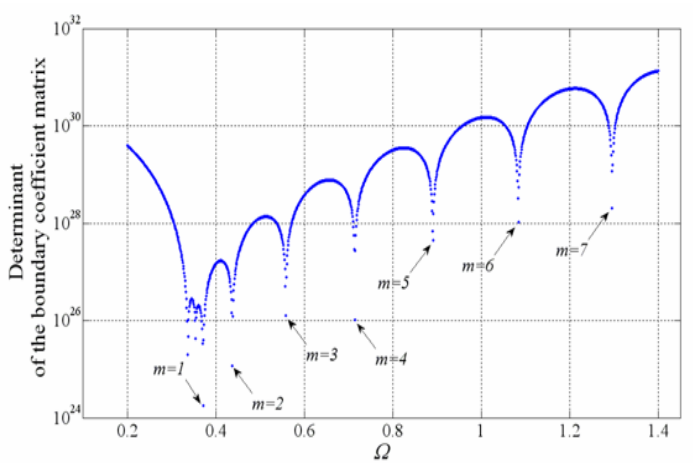

Fig. 5. Determinant of the boundary coefficient matrix versus frequency parameter for $n=5$.

points are observed. These minimum points are actual representations of the resonance frequencies of the shell, at which the determinants are zero. However, as discussed in section 2.2, the minimum points should also satisfy Eq. (13) in order to be the resonance frequency of the system. Thus, in Figs. 3-7, the unmarked minimum points before $m=7$ did not satisfy Eq. (13) and are not a resonance frequency.

Accordingly, at higher frequencies, higher axial wave parameters $(m)$ occur. Figs. 3-7 are graphical representations of how the exact resonance frequencies are obtained using the method described in this paper. According to the exact method, each minimum point in the diagrams represents a resonance frequency with a specific mode shape.

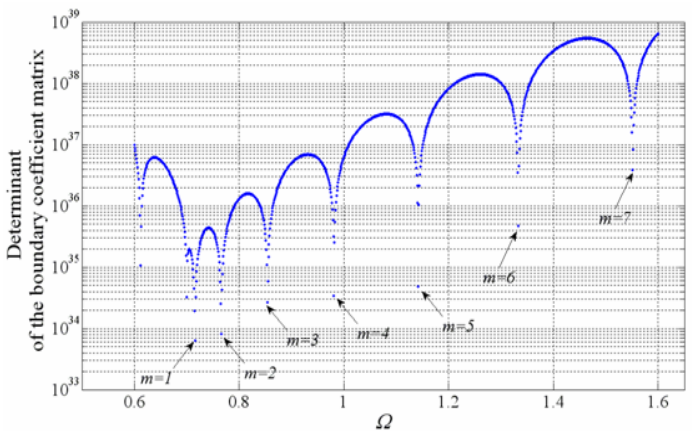

Fig. 6. Determinant of the boundary coefficient matrix versus frequency parameter for $n=7$.

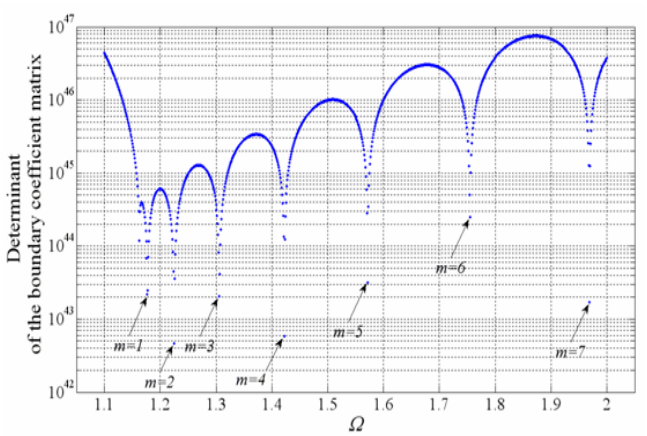

Fig. 7. Determinant of the boundary coefficient matrix versus frequency parameter for $n=9$.

Next, let us investigate the accuracy of using beam functions as an alternative to the exact method. To study such an approximation, natural frequencies were calculated using two methods: 1) the approximate method (in which $\lambda_{m}=$ $m \pi(R / L) \sqrt{-1}$ ) and 2) the exact method (Eq. (21)). In Tables $1-8$, the values of the non-dimensional frequency parameter $\Omega$ are compared for a simply supported cylindrical shell. Four diverse theories have been applied to evaluate their accuracy. All non-dimensional frequencies are calculated up to the fourth digit. As it can be seen, the approximate beam function analysis yields close results with the least errors. In Tables 1-8, mode shapes at which the approximate method obtains errors are marked by a $(*)$ sign.

As one can see from the comparisons of Tables 1-8, there are some minute discrepancies, which can be classified into two groups for all theories: first, those related to the $m=1$ modes, and second, the differences related to the $m \geq 2$ modes. In $m=1$ modes, the values of the errors are between 0.2-0.7 percent. However, for the $m \geq 2$ modes, the errors are between $0.001-0.01$ percent, which are mainly the result of a rounding error to the fourth digit. Moreover, according to Tables 1-8, as the axial wave parameter $m$ increases, the number of resonance frequencies containing an error decrease. Hence, the approximate method is similar to the exact analysis for $m \geq 2$ modes and for low mode numbers of $m=1$ with $n \geq 5$. On the other hand, for low mode numbers of $m=1$ with $1 \leq n \leq 4$, the approximate method yields high 
Table 1. Frequency parameter for the Soedel's shell theory by the approximation method with $L / R=3, h / R=1 / 20$ and $v=1 / 3$.

\begin{tabular}{c|c|c|c|c|c|c|c}
\hline$n$ & $m=1$ & $m=2$ & $m=3$ & $m=4$ & $m=5$ & $m=6$ & $m=7$ \\
\hline 1 & 0.3672 & 0.7045 & 0.8473 & 0.9213 & 0.9924 & 1.0859 & 1.2142 \\
2 & 0.1867 & 0.4647 & 0.6695 & 0.8097 & 0.9276 & 1.0533 & 1.2040 \\
3 & 0.1568 & 0.3384 & 0.5370 & 0.7098 & 0.8664 & 1.0258 & 1.2029 \\
4 & 0.2320 & 0.3339 & 0.4942 & 0.6682 & 0.8443 & 1.0275 & 1.2268 \\
5 & 0.3566 & 0.4233 & 0.5453 & 0.7029 & 0.8806 & 1.0748 & 1.2879 \\
6 & 0.5140 & 0.5683 & 0.6679 & 0.8075 & 0.9779 & 1.1732 & 1.3920 \\
7 & 0.7012 & 0.7510 & 0.8397 & 0.9670 & 1.1286 & 1.3203 & 1.5396 \\
8 & 0.9175 & 0.9656 & 1.0492 & 1.1691 & 1.3238 & 1.5109 & 1.7284 \\
9 & 1.1627 & 1.2102 & 1.2913 & 1.4070 & 1.5570 & 1.7402 & 1.9551 \\
10 & 1.4368 & 1.4840 & 1.5639 & 1.6772 & 1.8243 & 2.0044 & 2.2169 \\
\hline
\end{tabular}

Table 2. Frequency parameter for the Soedel's shell theory by the exact method with $L / R=3, h / R=1 / 20$ and $v=1 / 3$.

\begin{tabular}{c|c|c|c|c|c|c|c}
\hline$n$ & $m=1$ & $m=2$ & $m=3$ & $m=4$ & $m=5$ & $m=6$ & $m=7$ \\
\hline 1 & $0.3660^{*}$ & $0.7044^{*}$ & 0.8473 & 0.9213 & 0.9924 & 1.0859 & 1.2142 \\
2 & $0.1863^{*}$ & 0.4647 & 0.6695 & 0.8097 & 0.9276 & 1.0533 & 1.2040 \\
3 & $0.1520^{*}$ & $0.3382^{*}$ & 0.5370 & 0.7098 & 0.8664 & 1.0258 & 1.2029 \\
4 & 0.2320 & $0.3338^{*}$ & 0.4942 & 0.6682 & 0.8443 & 1.0275 & 1.2268 \\
5 & $0.3565^{*}$ & 0.4233 & 0.5453 & 0.7029 & 0.8806 & 1.0748 & 1.2879 \\
6 & 0.5140 & 0.5683 & 0.6679 & 0.8075 & 0.9779 & 1.1732 & 1.3920 \\
7 & 0.7012 & 0.7510 & 0.8397 & 0.9670 & 1.1286 & 1.3203 & 1.5396 \\
8 & $0.9174^{*}$ & $0.9655^{*}$ & $1.0491^{*}$ & $1.1690^{*}$ & 1.3238 & 1.5109 & 1.7284 \\
9 & 1.1627 & $1.2101^{*}$ & 1.2913 & $1.4069^{*}$ & 1.5570 & 1.7402 & $1.9550^{*}$ \\
10 & 1.4368 & 1.4840 & $1.5638^{*}$ & 1.6772 & $1.8242^{*}$ & 2.0044 & 2.2169 \\
\hline
\end{tabular}

Table 3. Frequency parameter for the Flugge's shell theory by the approximation method with $L / R=3, h / R=1 / 20$ and $v=1 / 3$.

\begin{tabular}{c|c|c|c|c|c|c|c}
\hline$n$ & $m=1$ & $m=2$ & $m=3$ & $m=4$ & $m=5$ & $m=6$ & $m=7$ \\
\hline 1 & 0.3676 & 0.7051 & 0.8479 & 0.9219 & 0.9929 & 1.0863 & 1.2147 \\
2 & 0.1907 & 0.4672 & 0.6718 & 0.8119 & 0.9297 & 1.0551 & 1.2057 \\
3 & 0.1680 & 0.3453 & 0.5425 & 0.7146 & 0.8708 & 1.0297 & 1.2064 \\
4 & 0.2458 & 0.3455 & 0.5037 & 0.6764 & 0.8515 & 1.0340 & 1.2325 \\
5 & 0.3709 & 0.4371 & 0.5578 & 0.7140 & 0.8906 & 1.0838 & 1.2959 \\
6 & 0.5285 & 0.5828 & 0.6818 & 0.8207 & 0.9900 & 1.1842 & 1.4020 \\
7 & 0.7158 & 0.7658 & 0.8543 & 0.9811 & 1.1421 & 1.3329 & 1.5514 \\
8 & 0.9322 & 0.9805 & 1.0641 & 1.1838 & 1.3382 & 1.5247 & 1.7414 \\
9 & 1.1775 & 1.2251 & 1.3064 & 1.4221 & 1.5719 & 1.7547 & 1.9691 \\
10 & 1.4517 & 1.4991 & 1.5791 & 1.6926 & 1.8395 & 2.0195 & 2.2316 \\
\hline
\end{tabular}

Table 4. Frequency parameter for the Flugge's shell theory by the exact method with $L / R=3, h / R=1 / 20$ and $v=1 / 3$.

\begin{tabular}{c|c|c|c|c|c|c|c}
\hline$N$ & $m=1$ & $m=2$ & $m=3$ & $m=4$ & $m=5$ & $m=6$ & $m=7$ \\
\hline 1 & $0.3649^{*}$ & $0.7050^{*}$ & 0.8479 & 0.9219 & 0.9929 & 1.0863 & 1.2147 \\
2 & $0.1900^{*}$ & 0.4672 & 0.6718 & 0.8119 & 0.9297 & 1.0551 & 1.2057 \\
3 & $0.1681^{*}$ & 0.3453 & 0.5425 & 0.7146 & 0.8708 & 1.0297 & 1.2064 \\
4 & $0.2457^{*}$ & 0.3455 & 0.5037 & 0.6764 & 0.8515 & 1.0340 & 1.2325 \\
5 & $0.3710^{*}$ & 0.4371 & 0.5578 & 0.7140 & 0.8906 & 1.0838 & 1.2959 \\
6 & 0.5285 & 0.5828 & 0.6818 & $0.8206^{*}$ & 0.9900 & 1.1842 & 1.4020 \\
7 & 0.7158 & 0.7658 & 0.8543 & 0.9811 & $1.1420^{*}$ & 1.3329 & $1.5513^{*}$ \\
8 & $0.9321^{*}$ & 0.9805 & 1.0641 & 1.1838 & $1.3381^{*}$ & $1.5246^{*}$ & 1.7414 \\
9 & 1.1775 & 1.2251 & $1.3063^{*}$ & $1.4220^{*}$ & 1.5719 & $1.7545^{*}$ & $1.9690^{*}$ \\
10 & 1.4517 & $1.4990^{*}$ & 1.5791 & $1.6925^{*}$ & 1.8395 & $2.0194^{*}$ & 2.2316 \\
\hline
\end{tabular}

errors and so is not accurate for low mode numbers. Thus, it should only be used for high mode numbers with high resonance frequencies.
Table 5. Frequency parameter for the Morley-Koiter's shell theory by the approximation method with $L / R=3, h / R=1 / 20$ and $v=1 / 3$.

\begin{tabular}{c|c|c|c|c|c|c|c}
\hline$n$ & $m=1$ & $m=2$ & $m=3$ & $m=4$ & $m=5$ & $m=6$ & $m=7$ \\
\hline 1 & 0.3673 & 0.7044 & 0.8467 & 0.9201 & 0.9905 & 1.0834 & 1.2112 \\
2 & 0.1865 & 0.4644 & 0.6688 & 0.8085 & 0.9258 & 1.0508 & 1.2010 \\
3 & 0.1565 & 0.3378 & 0.5361 & 0.7084 & 0.8645 & 1.0233 & 1.2000 \\
4 & 0.2318 & 0.3332 & 0.4932 & 0.6668 & 0.8424 & 1.0252 & 1.2240 \\
5 & 0.3565 & 0.4228 & 0.5443 & 0.7015 & 0.8788 & 1.0727 & 1.2853 \\
6 & 0.5140 & 0.5680 & 0.6671 & 0.8064 & 0.9764 & 1.1713 & 1.3897 \\
7 & 0.7013 & 0.7509 & 0.8392 & 0.9661 & 1.1274 & 1.3187 & 1.5377 \\
8 & 0.9177 & 0.9656 & 1.0489 & 1.1685 & 1.3229 & 1.5097 & 1.7268 \\
9 & 1.1630 & 1.2103 & 1.2912 & 1.4066 & 1.5564 & 1.7393 & 1.9539 \\
10 & 1.4372 & 1.4843 & 1.5640 & 1.6771 & 1.8239 & 2.0038 & 2.2160 \\
\hline
\end{tabular}

Table 6. Frequency parameter for the Morley-Koiter's shell theory by the exact method with $L / R=3, h / R=1 / 20$ and $v=1 / 3$.

\begin{tabular}{c|c|c|c|c|c|c|c}
\hline$n$ & $m=1$ & $m=2$ & $m=3$ & $m=4$ & $m=5$ & $m=6$ & $m=7$ \\
\hline 1 & $0.3653^{*}$ & $0.7043^{*}$ & 0.8467 & 0.9201 & 0.9905 & 1.0834 & 1.2112 \\
2 & $0.1870^{*}$ & 0.4644 & 0.6688 & 0.8085 & 0.9258 & 1.0508 & 1.2010 \\
3 & $0.1563^{*}$ & $0.3377^{*}$ & 0.5361 & 0.7084 & 0.8645 & 1.0233 & 1.2000 \\
4 & $0.2314^{*}$ & $0.3330^{*}$ & 0.4932 & $0.6667^{*}$ & $0.8423^{*}$ & $1.0251^{*}$ & 1.2240 \\
5 & $0.3564^{*}$ & $0.4227^{*}$ & 0.5443 & $0.7014^{*}$ & 0.8788 & $1.0726^{*}$ & 1.2853 \\
6 & 0.5140 & 0.5680 & 0.6671 & 0.8064 & $0.9763^{*}$ & $1.1712^{*}$ & 1.3897 \\
7 & 0.7013 & 0.7509 & 0.8392 & 0.9661 & $1.1273^{*}$ & 1.3187 & 1.5377 \\
8 & $0.9176^{*}$ & 0.9656 & 1.0489 & $1.1684^{*}$ & $1.3228^{*}$ & $1.5096^{*}$ & 1.7268 \\
9 & 1.1630 & 1.2103 & 1.2912 & 1.4066 & 1.5564 & $1.7392^{*}$ & $1.9538^{*}$ \\
10 & 1.4372 & 1.4843 & $1.5639^{*}$ & 1.6771 & 1.8239 & 2.0038 & 2.2160 \\
\hline
\end{tabular}

Table 7. Frequency parameter for the Donnell's shell theory by the approximation method with $L / R=3, h / R=1 / 20$ and $v=1 / 3$.

\begin{tabular}{c|c|c|c|c|c|c|c}
\hline$n$ & $m=1$ & $m=2$ & $m=3$ & $m=4$ & $m=5$ & $m=6$ & $m=7$ \\
\hline 1 & 0.3671 & 0.7039 & 0.8455 & 0.9179 & 0.9871 & 1.0787 & 1.2054 \\
2 & 0.1867 & 0.4643 & 0.6680 & 0.8067 & 0.9229 & 1.0467 & 1.1958 \\
3 & 0.1570 & 0.3382 & 0.5359 & 0.7074 & 0.8624 & 1.0201 & 1.1958 \\
4 & 0.2323 & 0.3341 & 0.4937 & 0.6666 & 0.8413 & 1.0231 & 1.2210 \\
5 & 0.3569 & 0.4237 & 0.5454 & 0.7021 & 0.8787 & 1.0717 & 1.2834 \\
6 & 0.5143 & 0.5688 & 0.6683 & 0.8075 & 0.9770 & 1.1712 & 1.3889 \\
7 & 0.7015 & 0.7516 & 0.8403 & 0.9673 & 1.1284 & 1.3193 & 1.5378 \\
8 & 0.9178 & 0.9662 & 1.0499 & 1.1697 & 1.3242 & 1.5108 & 1.7275 \\
9 & 1.1632 & 1.2108 & 1.2921 & 1.4079 & 1.5578 & 1.7406 & 1.9550 \\
10 & 1.4373 & 1.4847 & 1.5648 & 1.6783 & 1.8253 & 2.0053 & 2.2174 \\
\hline
\end{tabular}

Table 8. Frequency parameter for the Donnell's shell theory by the exact method with $L / R=3, h / R=1 / 20$ and $v=1 / 3$.

\begin{tabular}{c|c|c|c|c|c|c|c}
\hline$n$ & $m=1$ & $m=2$ & $m=3$ & $m=4$ & $m=5$ & $m=6$ & $m=7$ \\
\hline 1 & $0.3674^{*}$ & 0.7039 & 0.8455 & 0.9179 & 0.9871 & 1.0787 & 1.2054 \\
2 & $0.1858^{*}$ & $0.4642^{*}$ & 0.6680 & 0.8067 & 0.9229 & 1.0467 & 1.1958 \\
3 & $0.1563^{*}$ & $0.3381^{*}$ & 0.5359 & 0.7074 & 0.8624 & 1.0201 & $1.1957^{*}$ \\
4 & $0.2321^{*}$ & $0.3340^{*}$ & 0.4937 & 0.6666 & $0.8412^{*}$ & $1.0230^{*}$ & $1.2209^{*}$ \\
5 & $0.3568^{*}$ & $0.4236^{*}$ & $0.5453^{*}$ & 0.7021 & 0.8787 & $1.0716^{*}$ & 1.2834 \\
6 & 0.5143 & 0.5688 & 0.6683 & $0.8074^{*}$ & 0.9770 & 1.1712 & 1.3889 \\
7 & 0.7015 & $0.7515^{*}$ & 0.8403 & 0.9673 & 1.1284 & 1.3193 & $1.5377^{*}$ \\
8 & 0.9178 & 0.9662 & 1.0499 & 1.1697 & $1.3241^{*}$ & $1.5107^{*}$ & 1.7275 \\
9 & $1.1631^{*}$ & 1.2108 & $1.2920^{*}$ & $1.4078^{*}$ & $1.5577^{*}$ & 1.7406 & $1.9549^{*}$ \\
10 & 1.4373 & 1.4847 & $1.5647^{*}$ & $1.6782^{*}$ & $1.8252^{*}$ & $2.0052^{*}$ & 2.2174 \\
\hline
\end{tabular}

Comparing the different approximate theories listed in Tables 1-8, the approximate Soedel theory is the most accurate. In contrast, the approximate Morley-Koiter theory yields the 
highest errors. However, for all theories as the frequency and mode numbers increase, the errors of the approximation decrease.

The exact and approximate methods were applied to the four theories discussed: 1) Soedel, 2) Flugge, 3) MorleyKoiter and 4) Donnell. In Table 9, the exact and approximate results according to these theories are compared to an experiment held by Farshidianfar et al. [16]. The simply supported circular cylindrical shell investigated in Table 9 is made of aluminum with material properties of $E=68.2 \mathrm{GPa}$, $\rho=2700 \mathrm{Kg} / \mathrm{m}^{3}$ and $v=0.33$. The dimensions of the shell are $L=1.7272 \mathrm{~m}, R=0.0762 \mathrm{~m}$ and $h=0.00147 \mathrm{~m}$. Simulations were carried out using a finite element model (FEM). The finite element analysis was modeled in the commercial finite element analysis (FEA) software "ANSYS". The FEA was developed in the following order:

Pre-processing: includes defining the constructing mesh, elastic and strength properties, element type (Solid 45 for linear isotropic shell and Shell 99 for linear orthotropic shell), layer orientation, thickness for orthotropic shell, and geometrical modeling.

Processing: in this section, the finite element model of the modal analysis is proposed and constructed. Also, boundary condition effects (such as $v=w=0$ ) are applied to the boundary nodes.

Post-processing: this is the final step that presents the results according to the type of FEM analysis.

First, let us study the accuracy of the Soedel exact theory, since it is the main focus of this research. As shown in Table 9, the exact method applied to the Soedel theory is found be much more accurate than the approximate method. Interestingly, the exact method calculates the fundamental frequency $(m, n)=\{(1,1)\}$ with nearly no errors. Moreover, it is remarkable that the exact analysis predicts five resonance modes, $(m, n)=\{(1,1),(2,1),(2,3),(3,2),(5,3)\}$, with errors of equal or less than one percent. Although the errors of the exact method are higher than those of the approximate at some resonance frequencies, the difference in the errors are small and negligible. However, the exact analysis has reduced the errors of most resonance frequencies dramatically. For example, at mode shapes of $(m, n)=\{(2,1),(3,2)\}$, the 13 percent error of the approximate theory is reduced to less than 1 percent using the exact analysis. On the other hand, some errors exist at low mode numbers for both the exact and approximate methods, especially for $(m, n)=\{(2,2)\}$. However, as the frequency increases, the errors decrease. Thus, at high mode numbers, the exact method of the Soedel theory is in complete agreement with experimental and numerical results. Such a trend is also observed for the approximate method; however, with higher errors at low mode numbers. According to Tables 9 and 10 , the Flugge theory is also very similar to the Soedel theory, both in terms of accuracy and errors. Although at low mode numbers the errors of the Flugge theory are higher than those of the Soedel's, the exact results of this theory at high mode numbers have less errors. For example, in modes
Table 9. Comparison between exact and approximation analysis with experimental and numerical data (FEM).

\begin{tabular}{|c|c|c|c|c|c|c|c|c|c|c|c|}
\hline$m$ & $n$ & 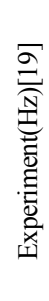 & 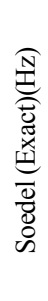 & 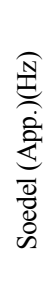 & 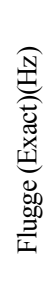 & 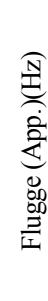 & 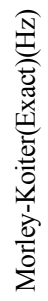 & 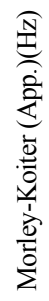 & 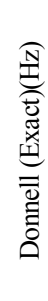 & 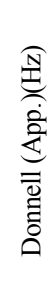 & 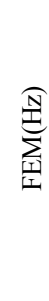 \\
\hline 1 & 1 & 138 & 139 & 141 & 140 & 142 & 134 & 142 & 101 & 149 & 135 \\
\hline 1 & 2 & 190 & 172 & 177 & 169 & 177 & 164 & 177 & 219 & 232 & 184 \\
\hline 1 & 3 & 502 & 482 & 482 & 552 & 489 & 406 & 482 & 545 & 542 & 512 \\
\hline 1 & 4 & 884 & 920 & 922 & 920 & 922 & 928 & 922 & 981 & 984 & 998 \\
\hline 2 & 1 & 465 & 467 & 527 & 506 & 528 & 543 & 528 & 543 & 532 & 505 \\
\hline 2 & 2 & 310 & 243 & 250 & 253 & 250 & 273 & 250 & 321 & 292 & 312 \\
\hline 2 & 3 & 477 & 482 & 492 & 481 & 492 & 406 & 492 & 545 & 551 & 521 \\
\hline 3 & 2 & 497 & 494 & 432 & 440 & 432 & 441 & 433 & 516 & 459 & 511 \\
\hline 3 & 3 & 559 & 491 & 525 & 586 & 525 & 596 & 526 & 545 & 582 & 551 \\
\hline 4 & 2 & 680 & 647 & 701 & 688 & 701 & 675 & 703 & 685 & 719 & 677 \\
\hline 4 & 3 & 638 & 575 & 599 & 669 & 598 & 645 & 599 & 650 & 650 & 618 \\
\hline 5 & 3 & 782 & 789 & 720 & 738 & 720 & 741 & 721 & 763 & 764 & 730 \\
\hline
\end{tabular}

Table 10. Errors of the exact and approximate methods with respect to experimental data.

\begin{tabular}{|c|c|c|c|c|c|c|c|c|c|c|}
\hline$m$ & $n$ & $\begin{array}{c}\text { Soedel } \\
\text { Exact) } \\
(\%)\end{array}$ & $\begin{array}{c}\text { Soedel } \\
\text { (App.) } \\
(\%)\end{array}$ & $\begin{array}{c}\text { Flugge } \\
(\text { Exact }) \\
(\%)\end{array}$ & $\begin{array}{c}\text { Flugge } \\
(\text { App.) } \\
(\%)\end{array}$ & \begin{tabular}{|c|} 
Morley \\
- Koiter \\
$($ Exact $)$ \\
$(\%)$
\end{tabular} & $\begin{array}{c}\text { Morley } \\
- \text {-Koiter } \\
(\text { App. }) \\
(\%)\end{array}$ & $\begin{array}{c}\text { Donnell } \\
(\text { Exact) } \\
(\%)\end{array}$ & $\begin{array}{c}\text { Donnell } \\
\text { (App.) } \\
(\%)\end{array}$ & $\begin{array}{c}\text { FEM } \\
(\%)\end{array}$ \\
\hline 1 & 1 & 0.8 & 2.2 & 1. & 3 & -3.3 & 2 & -27.1 & 7.4 & -2.5 \\
\hline 1 & 2 & -9.4 & -7.1 & -10.9 & -7.2 & -6.1 & -7.1 & 15.0 & 21.8 & -3.1 \\
\hline 1 & 3 & -4.1 & -4.1 & 9.8 & -4.1 & -19.2 & -4.0 & 8.5 & 7.9 & 1.9 \\
\hline 1 & 4 & 4.0 & 4.3 & 4.0 & 4.3 & 4.9 & & 10.9 & -11.2 & 12.8 \\
\hline 2 & 1 & 0.5 & 13.5 & 8.9 & 13.6 & 16.7 & 13.6 & 16.9 & 14.5 & 8.7 \\
\hline 2 & 2 & -21.9 & -19.6 & -18.3 & -19.6 & -12.2 & -19.5 & 3.5 & -6.0 & 0.5 \\
\hline 2 & 3 & 1.0 & 3.1 & 0.9 & 3.1 & -14.9 & 3.2 & 15.6 & 14.2 & 9.3 \\
\hline 3 & 2 & -0.4 & -13.0 & -11.3 & -13.0 & -11.1 & -12.8 & 3.9 & -7.6 & 3.0 \\
\hline 3 & 3 & -12.1 & -6.0 & 4.9 & -6.0 & 6.6 & -5.9 & -2.5 & 4.2 & -1.4 \\
\hline 4 & 2 & -4.8 & 3.1 & 1.2 & 3.2 & -0.8 & 3.2 & 0.7 & 5.7 & -0.4 \\
\hline 4 & 3 & -9.9 & -6.2 & 4.8 & 6.2 & 1.0 & 6.1 & 1.7 & 1.8 & -3.2 \\
\hline 5 & 3 & 0.9 & -7.9 & -5.7 & -7.9 & -5.2 & -7.7 & -2.4 & -2.2 & -6.6 \\
\hline
\end{tabular}

$(m, n)=\{(3,3),(4,2)\}$, the exact Flugge theory obtains less errors compared to the Soedel theory. Moreover, comparing results of the exact and approximate methods in the Flugge theory with experimental results, it is observed that the exact method is more accurate in nearly all frequency ranges. Thus, the exact method is a good choice when applied to the Flugge theory. On the other hand, a rather different behavior is observed for the Morley-Koiter and Donell theories. The exact method of the Morley-Koiter theory has high errors in most mode shapes, especially at low mode numbers. Hence, an exact analysis of this theory may not be suitable. The exact results of the Donell theory are more accurate than the approximate method; however, there are a few modes at which the errors increase slightly. The only high error is yielded at mode $(m, n)=\{(1,1)\}$, in which the error is increased dramatically to 21.7 percent. In conclusion, the exact method im- 

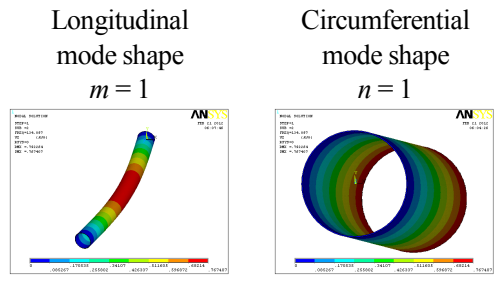

(a)
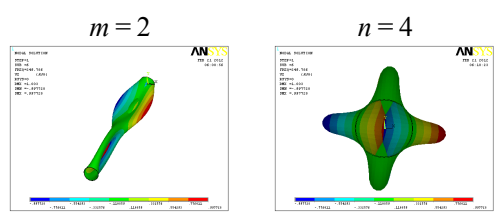

(b)
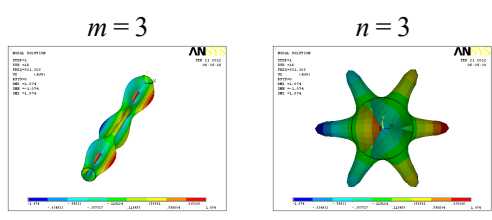

(c)
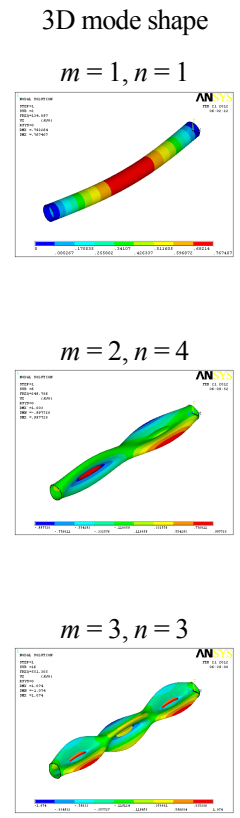

Fig. 8. Mode shapes of the circular cylindrical shell: (a) for $m=1, n=$ ; (b) for $m=2, n=4$; (c) for $m=3, n=3$.

proved results of the approximate technique compared to experimental and numerical data. This statement is correct for all theories, except for the Morley-Koiter. The accuracy of the exact method increased at high mode numbers. Generally, the Soedel and Flugge theories yielded the most accurate results compared to experimental data, predicting accurate resonance frequencies in low and high mode numbers.

The FEM results are found to be in strong agreement with the experimental ones as well. Of note, at some mode shapes the FEM is even more accurate than the other theories discussed. In Fig. 8, mode shapes are reported from the finite element analysis for $(m, n)=\{(1,1),(2,4),(3,3)\}$ modes.

Overall, the validity of the exact method was approved with comparison to experimental, numerical and theoretical results. The approximate method was shown to be imprecise at low mode numbers. However, at high mode numbers the approximate technique was in good agreement with experimental and exact results.

\section{Conclusions}

The free vibration of circular cylindrical shells with simply supported boundary conditions has been studied using four different thin shell theories: Soedel, Flugge, Morley-Koiter and Donnell. The scope of the investigation was focused upon the exact analysis of natural frequencies. The approximate beam function method was also evaluated. First, a graphical representation of the exact analysis was presented in order to find the natural frequencies of a shell. Next, exact results of the four theories were compared to approximate calculations.

It was observed that, for low mode numbers, the approximate method yields different results than the exact method does, whereas, for high mode numbers, no significant discrepancies were noticed. Moreover, the approximate method based on the Soedel theory obtained better results than the other theories. To check the accuracy of the exact method, experimental and numerical results were compared. According to this comparison, the exact analysis predicted most of the resonance frequencies with errors of less than one percent. In contrast, the approximate method yielded high errors for some mode shapes.

\section{References}

[1] W. Leissa, Vibration of shells, US Government Printing Office, Washington DC, USA (1973).

[2] M. Amabili and M. P. Paidoussis, Review of studies on geometrically nonlinear vibrations and dynamics of circular cylindrical shells and panels, with and without fluid structure interaction, Applied Mechanics Reviews, 56 (2003) 349-381.

[3] M. Amabili, Nonlinear vibrations and stability of shells and plates, Cambridge University Press, New York, USA (2008).

[4] Y. Kurylov and M. Amabili, Polynomial versus trigonometric expansions for nonlinear vibrations of circular cylindrical shells with different boundary conditions, Journal of Sound and Vibration, 329 (2010) 1435-1449.

[5] W. Flugge, Stresses in shells, Springer, New York, USA (1973).

[6] A. E. H. Love, On the small free vibrations and deformations of thin shells, Philosophical Transactions of the Royal Society, 179A (1888) 491-546.

[7] K. K. Livanov, Axisymmeric vibrations of simply supported cylindrical shells, PMM, 25 (1961) 742-745.

[8] S. A. Rinehart and J. T. S. Wang, Vibration of simply supported cylindrical shells with longitudinal stiffeners, Journal of Sound and Vibration, 24 (1972) 151-163.

[9] G. B. Warburton and J. Higgs, Natural frequencies of thin cantilever cylindrical shells, Journal of Sound and Vibration, 11 (1970) 335-338.

[10] C. B. Sharma, Calculation of natural frequencies of fixedfree circular cylindrical shells, Journal of Sound and Vibration, 35 (1974) 55-76.

[11] J. Callahan and H. Baruh, A closed-form solution procedure for circular cylindrical shell vibrations, International Journal of Solids and Structures, 36 (1999) 2973-3013.

[12] F. Moussaoui, R. Benamar and R. G. White, The effects of large vibration amplitudes on the mode shapes and natural frequencies of thin elastic shells, Part I: Coupled transversecircumferential mode shapes of isotropic circular cylindrical shells of infinite length, Journal of Sound and Vibration, 232 (2000) 917-943.

[13] J. Lee, Free vibration analysis of a hermetic capsule by pseudospectral method, Journal of Mechanical Science and Technology, 26 (4) (2012) 1011-1015.

[14] W. Soedel, Vibrations of shells and plates, $3^{\text {rd }}$ ed., Marcel 
Dekker, New York, USA (2004).

[15] J. Callahan, Cylindrical shell vibrations: Closed-form analysis and measurement via piezoelectric films, PHD Dissertation, New Brunswick Rutgers, The State University of New Jersey, USA (1997).

[16] A. Farshidianfar, M. H. Farshidianfar, M. J. Crocker and W. O. Smith, The vibration analysis of long cylindrical shells using acoustical excitation, Journal of Sound and Vibration, 232 (2010) 917-943.

\section{Appendix A}

\section{A.1 Differential operators according to the Donnell theory}

$$
\mathbf{L}_{\mathbf{D}}=\left[\begin{array}{ccc}
-\frac{\rho}{E}\left(1-v^{2}\right) \frac{\partial^{2}}{\partial t^{2}} & \frac{1+v}{2 R} \frac{\partial^{2}}{\partial x \partial \theta} & \frac{v}{R} \frac{\partial}{\partial x} \\
+\frac{\partial^{2}}{\partial x^{2}}+\frac{1-v}{2 R^{2}} \frac{\partial^{2}}{\partial \theta^{2}} & -\frac{\rho}{E}\left(1-v^{2}\right) \frac{\partial^{2}}{\partial t^{2}} & \frac{1}{R^{2}} \frac{\partial}{\partial \theta} \\
\frac{1+v}{2 R} \frac{\partial^{2}}{\partial x \partial \theta} & +\frac{1-v}{2} \frac{\partial^{2}}{\partial x^{2}}+\frac{1}{R^{2}} \frac{\partial^{2}}{\partial \theta^{2}} & -\frac{\rho}{E}\left(1-v^{2}\right) \frac{\partial^{2}}{\partial t^{2}} \\
-\frac{v}{R} \frac{\partial}{\partial x} & -\frac{1}{R^{2}} \frac{\partial}{\partial \theta} & -\left[\frac{1}{R^{2}}+k\left(R \frac{\partial^{2}}{\partial x^{2}}+\frac{1}{R} \frac{\partial^{2}}{\partial \theta^{2}}\right)^{2}\right.
\end{array}\right]
$$

\section{A.2 Modifying differential operators according to the Soedel} theory

$$
\mathbf{L}_{\mathbf{M O D}}=\left[\begin{array}{ccc}
0 & 0 & 0 \\
0 & +\frac{1-v}{2} \frac{\partial^{2}}{\partial x^{2}}+\frac{1}{R^{2}} \frac{\partial^{2}}{\partial \theta^{2}} & -\frac{\partial^{2}}{\partial x^{2}}+\frac{1}{R^{2}} \frac{\partial^{2}}{\partial \theta^{2}} \\
0 & \frac{\partial^{2}}{\partial x^{2}}+\frac{1}{R^{2}} \frac{\partial^{2}}{\partial \theta^{2}} & 0
\end{array}\right]
$$

A.3 Modifying differential operators according to the Flugge theory

$$
\mathbf{L}_{\text {MOD }}=\left[\begin{array}{ccc}
+\frac{1-v}{2 R^{2}} \frac{\partial^{2}}{\partial \theta^{2}} & 0 & \frac{1-v}{2 R} \frac{\partial^{3}}{\partial x \partial \theta^{2}}-R \frac{\partial^{3}}{\partial x^{3}} \\
0 & \frac{3(1-v)}{2} \frac{\partial^{2}}{\partial x^{2}} & -\left(\frac{3-v}{2}\right) \frac{\partial^{3}}{\partial x^{2} \partial \theta} \\
-\frac{1-v}{2 R} \frac{\partial^{3}}{\partial x \partial \theta^{2}}+R \frac{\partial^{3}}{\partial x^{3}} & \left(\frac{3-v}{2}\right) \frac{\partial^{3}}{\partial x^{2} \partial \theta} & -\frac{1}{R^{2}}\left(1+\frac{2 \partial^{2}}{\partial \theta^{2}}\right)
\end{array}\right]
$$

A.4 Modifying differential operators according to the Morley-Koiter theory

$$
\mathbf{L}_{\text {MOD }}=\left[\begin{array}{ccc} 
& & 0 \\
0 & 0 & 0 \\
0 & 0 & 1 \\
0 & 0 & -\frac{1}{R^{2}}\left(2 \frac{\partial^{2}}{\partial \theta^{2}}+1\right)-2 \frac{\partial^{2}}{\partial x^{2}}
\end{array}\right]
$$

\section{Appendix B}

\section{B.1 Coefficient matrix according to the Soedel theory}

$$
\left[\begin{array}{ccc}
\Omega^{2}+\lambda_{m}^{2}-\frac{1-v}{2} n^{2} & -\frac{1+v}{2} n \lambda_{m} & v \lambda_{m} \\
\frac{1+v}{2} n \lambda_{m} & \Omega^{2}+(1+k)\left(\frac{1-v}{2} \lambda_{m}^{2}-n^{2}\right) & n\left[1+k\left(n^{2}-\lambda_{m}^{2}\right)\right] \\
-v \lambda_{m} & n\left[1+k\left(n^{2}-\lambda_{m}^{2}\right)\right] & \Omega^{2}-\left[1+k\left(\lambda_{m}^{2}-n^{2}\right)^{2}\right]
\end{array}\right]
$$

\section{B.2 Coefficient matrix according to the Flugge theory}

$$
\left[\begin{array}{ccc}
\Omega^{2}+\lambda_{m}^{2}-n^{2}(1+k)\left(\frac{1-v}{2}\right) & -\frac{1+v}{2} n \lambda_{m} & \nu \lambda_{m}-k \lambda_{m}\left(\frac{1-v}{2} n^{2}+\lambda_{m}^{2}\right) \\
\frac{1+v}{2} n \lambda_{m} & \Omega^{2}+(1+3 k)\left(\frac{1-v}{2} \lambda_{m}^{2}\right)-n^{2} & n\left(1-\frac{3-v}{2} k \lambda_{m}^{2}\right) \\
-v \lambda_{m}+k \lambda_{m}\left(\frac{1-v}{2} n^{2}+\lambda_{m}^{2}\right) & n\left(1-\frac{3-v}{2} k \lambda_{m}^{2}\right) & \Omega^{2}-\left[1+k+k\left(\lambda_{m}^{2}-n^{2}\right)^{2}-2 k n^{2}\right]
\end{array}\right]
$$

\section{B.3 Coefficient matrix according to the Morley-Koiter theory}

$$
\left[\begin{array}{ccc}
\Omega^{2}+\lambda_{m}^{2}-n^{2}\left(\frac{1-v}{2}\right) & -\frac{1+v}{2} n \lambda_{m} & v \lambda_{m} \\
\frac{1+v}{2} n \lambda_{m} & \Omega^{2}+\left(\frac{1-v}{2} \lambda_{m}^{2}\right)-n^{2} & n \\
-v \lambda_{m} & n & \Omega^{2}-\left[1+k+k\left(\lambda_{m}^{2}-n^{2}\right)\left(\lambda_{m}^{2}-n^{2}+2\right)\right]
\end{array}\right]
$$

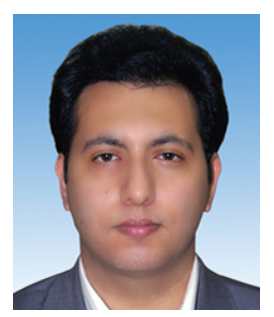

Pouria Oliazadeh received his B.S. and M.S. degrees from Ferdowsi University of Mashhad in 2010 and 2012, respectively and now he is the student of Ph.D. in Ferdowsi University of Mashhad. His research interests include: vibration, dynamics, acoustics and composite structures.

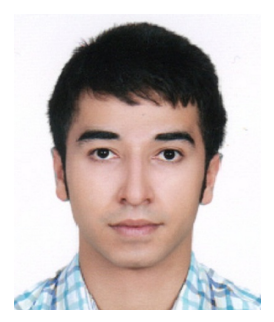

Mohammad H. Farshidianfar received the B.S. degree in mechanical engineering from Ferdowsi University of Mashhad, Mashhad, Iran in 2012. He is currently pursuing the M.A.Sc. degree in mechanical engineering at University of Waterloo, Waterloo, Canada. His research interests include: vibration, dynamics, acoustics and control. Mr. Farshidianfar has been the subject of several awards.

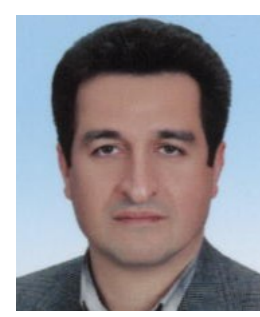

Anooshiravan Farshidianfar received the B.S. and M.S. degrees in mechanical engineering from University of Tehran, Tehran, Iran. He received the Ph.D. in mechanical engineering from University of Bradford, Bradford, England, in 2000. He is currently a Professor at Ferdowsi University of Mashhad, Mashhad, Iran. The current research interests in Professor Farshidianfar's group include: vibration, dynamics, acoustics and nano technology. Professor Farshidianfar has authored and co-authored over 180 publications. 